\title{
Tuberculosis Screening Before and During Treatment with Tumor Necrosis Factor Antagonists: Something Old, Something New
}
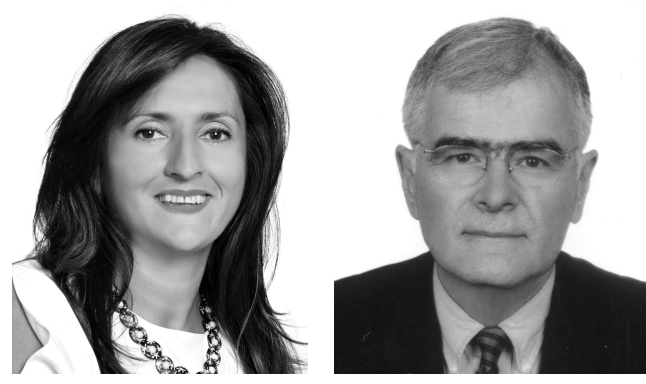

The increased risk of tuberculosis (TB) with tumor necrosis factor $(\mathrm{TNF}-\alpha)$ antagonist treatment is well recognized ${ }^{1}$. The risk seems to parallel the background risk of TB. In Spain, where the incidence in the general population is $23 / 100,000$, the incidence risk ratio of TB was 4.13 (95\% CI 2.59-6.83) in patients with rheumatoid arthritis (RA) compared to the general population and 19.9 (95\% CI 16.2-24.8) in patients with RA who were exposed to TNF- $\alpha$ antagonists compared to those who were not. In Sweden, where the incidence is $5 / 100,000$, the incidence risk ratio was 2.0 (95\% CI 1.2-3.4) in patients with RA and 4.0 (95\% CI 1.3-12) in patients with RA using TNF- $\alpha$ antagonists ${ }^{1,2}$. In more than half of the patients, TB during TNF- $\alpha$ antagonist use is extrapulmonary and/or disseminated ${ }^{1}$. There seems to be a bimodal pattern with reactivation of latent TB early during the treatment course, with primary active TB usually developing later in the course. After the implementation of routine screening programs, with increased awareness of the clinical and radiological clues along with use of the tuberculin skin test (TST) and interferon- $\gamma$ (IFN- $\gamma$ ) release assays (IGRA), a significant decrease in TB reactivation was observed among patients using TNF- $\alpha$ antagonists $^{3}$. However, there is still no consensus on whether TST or IGRA should be preferred for screening in these patients, who are usually immunocompromised because of the character of their diseases and the immunosuppressives they use. In this issue of The Journal, Costantino, et $a l^{4}$ report the results of TST and T-SPOT.TB assay in a large cohort of patients who are candidates for TNF- $\alpha$ antagonist therapy.

The TST is a time-honored method of showing that one has been exposed to mycobacteria. Its potential shortcomings are giving false-positive results because of bacillus Calmette-Guerin (BCG) vaccination and infection with other mycobacteria, and false-negative results because of immunosuppression and old age. Standardization of the performance and reading of the test may also be a problem. IGRA were developed to overcome these shortcomings and replace the TST for screening latent TB. There are 2 types of commercially available IGRA: the QuantiFERON-TB Gold (QFT-G) In-Tube test and TSPOT.TB. QFT-G and the older versions, Quantiferon and Quantiferon-TB Gold, measure antigen-specific IFN- $\gamma$ released by circulating $\mathrm{T}$ cells in whole blood. On the other hand, T-SPOT.TB measures the presensitized $\mathrm{T}$ cells specific to Mycobacterium tuberculosis isolated from peripheral blood mononuclear cells, which release IFN- $\gamma$.

Both of these methods were developed with the hope that they would be more reliable than TST because they are not affected by either BCG vaccination or infections with other mycobacteria, and they would perform better in immunosuppressed patients. These contentions were later challenged by several studies. It was reported that patients with $M$. leprae, M. avium, M. intracellulare, M. marinum, and $M$. kansasii may test positive with $\mathrm{QFT}^{5} \mathrm{G}^{5,6}$. A metaanalysis reported significant within-subject variability for IGRA, especially when values are close to the cutoff point ${ }^{7}$. Testing of immunosuppressed populations presented a problem with IGRA, too. A metaanalysis of studies conducted in human immunodeficiency virus (HIV)-infected individuals showed that the IGRA have a similar performance to TST in identifying latent $\mathrm{TB}^{8}$.

Several studies have been published comparing the sensitivity and specificity of TST with IGRA in different populations. In a metaanalysis of studies where active TB was used as a surrogate for latent TB, the pooled sensitivity was $0.78(0.73-0.82)$ for QFT-G, $0.90(0.86-0.93)$ for ELISPOT, and $0.77(0.71-0.82)$ for TST. The specificity was estimated as $0.96(0.94-0.98)$ for QFT-G, 0.93 (0.86-1.00) for ELISPOT, and 0.59 (0.46-0.73) for TST in BCG-vaccinated patients and $0.99(0.98-1.00)$ for QFT-G and $0.97(0.95-0.99)$ for TST with no BCG vaccination 9 The major problem with studies comparing the performance of TST with IGRA is the lack of a gold standard for defining latent TB. Moreover, the populations tested show wide variation, including such diverse groups as healthcare workers, immigrants, immunocompromised individuals with HIV, patients with endstage renal disease, or as in this

See LTBI screening in chronic inflammatory arthritis, page 1986 
study, candidates for TNF- $\alpha$ antagonist treatment. Thus, bringing together or comparing the sensitivities and specificities reported in different studies is not reliable. Moreover, the positive and negative predictive values, what one really needs to know before a test, would differ in each country owing to differences in the TB rate in the general population.

Other investigators have also tested the performance of IGRA for latent TB screening before TNF- $\alpha$ antagonist use $^{10,11,12,13,14,15,16}$. The common finding in these studies has been the low concordance between TST and IGRA results. There was a high false positivity rate with TST and more discordant results in BCG-vaccinated patients. Also, the frequency of indeterminate results with IGRA was high, usually associated with background immunosuppressive use and high C-reactive protein levels. The results and the interpretation of these studies show variation depending on the country, TB prevalence, income of the country, and characteristics of the patients who are included such as their diagnosis, disease severity, age, and concomitant and previous corticosteroid and immunosuppressive use. Some researchers have suggested replacing TST with IGRA, while others disagree ${ }^{10,11}$. A 2-step strategy in which an IGRA is used to confirm positive TST results or when false-negative TST results are suspected has also been proposed ${ }^{12}$. Still others suggest that especially in high-risk populations, a positive result with any of these methods warrants treatment for latent $\mathrm{TB}$ infection (LTBI) ${ }^{13,14}$. There is also no consensus on the cost-effectiveness of these strategies in studies from different countries ${ }^{15,16}$. Thus, it is hard to draw conclusions by generalizing the results obtained from these studies. Consideration of individual factors becomes more important, including history, clinical and radiographic findings, and whether prophylaxis should be started.

When recommending one screening method, the risk of the possibility of treating an unnecessary number of patients with a suspicion of LTBI needs to be weighed against the risk of activation of LTBI, which may follow a very severe course. Costantino, et $a l^{4}$ started anti-TB chemoprophylaxis if the patient had 1 or more clinical risk factors for latent TB or either TST or T-SPOT.TB positivity. This resulted in prescribing chemoprophylaxis in more than half of their patients. None of their patients developed TB during the 5 years of followup. If they were to use only T-SPOT.TB instead of both TST and T-SPOT.TB, 27\% of their patients would be spared using chemoprophylaxis. However, would this be safe?

Longterm followup studies of individuals who tested positive with either of these tests, and for some reason did not receive LTBI treatment, could shed light on this question. A systematic review of longitudinal studies in which IGRA were used either instead of TST or to confirm a positive TST result identified 7 such studies from high-income countries and 4 from low-income countries ${ }^{17}$.
Among the surveys from the high-income countries, an increase in incident TB was observed in only 1 survey. It was from the Netherlands, and tested immigrant TB contacts born in high-burden countries. The high-income countries were those with a low background TB prevalence, such as Germany, Switzerland, the Netherlands, Italy, and Norway; an exception was South Korea. Among the surveys from middle-income and low-income countries, there was an increase in incident TB with this approach in 2 of the 4 studies. One of these was from Gambia, with an increase of 0.08 . Another was from South Africa, with an increase of 0.03 per 100 patient-years. One can assume that this increase would be higher if these individuals had received TNF- $\alpha$ antagonists. On the other hand, another study from Korea, looking at TB rates in TST-negative/IGRA-positive kidney transplant recipients who were not treated for LTBI, reported that 4/71 such patients developed TB after kidney transplantation $^{18}$. Thus, based on the available data, in low-prevalence populations it seems safe and more practical to prefer one of the IGRA to TST for screening before prescribing TNF- $\alpha$ antagonists. However, in high-prevalence populations and in high-risk individuals, a positive result with any of the methods discussed seems to warrant treatment for latent TB. We reason that a 2-step strategy, involving performing a TST and treating patients over 5 $\mathrm{mm}$, not treating patients between 1-4 $\mathrm{mm}$, and performing an IGRA to those unresponsive to TST, could be used in high-risk settings.

Another consideration is whether it would be better to evaluate IGRA results quantitatively instead of qualitatively. The amount of IFN- $\gamma$ produced by the patient in response to mycobacterial antigens as well as that in response to mitogen (the positive control) gives important information. For example, in a patient who tested positive with the QFT-G test, with an IFN- $\gamma$ response just over the cutoff point, and assuming the IFN- $\gamma$ response to the positive control is significantly high, there is a possibility that the result may be false-positive. On the other hand, in a patient with a negative result, if the positive control IFN- $\gamma$ is also low - but not low enough to give an indeterminate result - the possibility of a false-negative result needs to be considered, especially if the patient is immunosuppressed. Studies have commented on the need for redefining cutoffs, but regardless of the cutoff level to call a patient positive, it seems wise to pay attention to the actual levels of IFN- $\gamma$ in response to mycobacterial antigens and mitogen when making a clinical decision.

Apart from screening for latent TB, monitoring of these patients for the development of new TB is also a problem. A concern was whether treatment with TNF- $\alpha$ antagonists would hamper the usefulness of TST and IGRA because of a reduced IFN- $\gamma$ response observed in such patients. Our group and others have shown that both TST and IGRA do not seem to be affected by TNF- $\alpha$ antagonists $6,19,20$.

Personal non-commercial use only. The Journal of Rheumatology Copyright @ 2013 . All rights reserved. 
However, there was a high frequency of reversions and conversions with both tests, probably related to reproducibility issues and changes in the immune status of the patient during treatment. Because the problem here is active TB, which would have clinical and laboratory signs unlike in latent TB, it is probably not useful to monitor patients with TST or IGRA during treatment. The usefulness of these tests when active TB is suspected is another problem, because of a high rate of false-negatives in active TB with either test.

The decision to use TST and/or IGRA for screening before TNF- $\alpha$ antagonists and the interpretation of the results of each test should be made individually in every patient, considering his/her disease type, previous medications, prevalence of TB in the geographical and social setting to which the patient belongs, and the actual INF- $\gamma$ levels for IGRA. Development of new IGRA, which we understand is already under way, may overcome some of these problems by providing better diagnostic accuracy ${ }^{21}$.

\author{
GULEN HATEMI, MD, Associate Professor; \\ HASAN YAZICI, MD, Professor; \\ Istanbul University, \\ Cerrahpasa Medical School, \\ Department of Internal Medicine, \\ Division of Rheumatology, \\ Istanbul, Turkey
}

Address correspondence to Dr. G. Hatemi, Associate Professor, Istanbul University, Cerrahpasa Medical School, Department of Internal Medicine, Division of Rheumatology, Istanbul 34300, Turkey.

E-mail: gulenhatemi@yahoo.com

\section{REFERENCES}

1. Gómez-Reino JJ, Carmona L, Valverde VR, Mola EM, Montero MD; BIOBADASER Group. Treatment of rheumatoid arthritis with tumor necrosis factor inhibitors may predispose to significant increase in tuberculosis risk: a multicenter active-surveillance report. Arthritis Rheum 2003;48:2122-7.

2. Askling J, Fored CM, Brandt L, Baecklund E, Bertilsson L, Cöster $\mathrm{L}$, et al. Risk and case characteristics of tuberculosis in rheumatoid arthritis associated with tumor necrosis factor antagonists in Sweden. Arthritis Rheum 2005;52:1986-92.

3. Carmona L, Gómez-Reino JJ, Rodríguez-Valverde V, Montero D, Pascual-Gómez E, Mola EM, et al; BIOBADASER Group. Effectiveness of recommendations to prevent reactivation of latent tuberculosis infection in patients treated with tumor necrosis factor antagonists. Arthritis Rheum 2005;52:1766-72.

4. Costantino F, de Carvalho Bittencourt M, Rat A-C, Loeuille D, Dintinger H, Béné MC, et al. Screening for latent tuberculosis infection in patients with chronic inflammatory arthritis: discrepancies between tuberculin skin test and interferon- $\gamma$ release assay results. J Rheumatol 2013;40:1986-93.

5. Kobashi Y, Mouri K, Yagi S, Obase Y, Miyashita N, Okimoto N, et al. Clinical evaluation of the QuantiFERON-TB Gold test in patients with non-tuberculous mycobacterial disease. Int J Tuberc Lung Dis 2009;13:1422-6.

6. Hatemi G, Melikoglu M, Ozbakir F, Tascilar K, Yazici H. Quantiferon-TB Gold in tube assay for the screening of tuberculosis before and during treatment with tumor necrosis factor-alpha antagonists. Arthritis Res Ther 2012;14:R147.
7. vanZyl-Smit RN, Zwerling A, Dheda K, Pai M. Within-subject variability of interferon-g assay results for tuberculosis and boosting effect of tuberculin skin testing: a systematic review. PLoS One 2009;4:e8517.

8. Santin M, Muñoz L, Rigau D. Interferon- $\gamma$ release assays for the diagnosis of tuberculosis and tuberculosis infection in HIV-infected adults: a systematic review and meta-analysis. PLoS One 2012;7:e32482.

9. Pai M, Zwerling A, Menzies D. Systematic review: T-cell-based assays for the diagnosis of latent tuberculosis infection: an update. Ann Intern Med 2008;149:177-84.

10. Mariette X, Baron G, Tubach F, Lioté F, Combe B, Miceli-Richard $\mathrm{C}$, et al. Influence of replacing tuberculin skin test with exvivo interferon $\gamma$ release assays on decision to administer prophylactic antituberculosis antibiotics before anti-TNF therapy. Ann Rheum Dis 2012;71:1783-90.

11. Greenberg JD, Reddy SM, Schloss SG, Kurucz OS, Bartlett SJ, Abramson SB, et al. Comparison of an in vitro tuberculosis interferon-gamma assay with delayed-type hypersensitivity testing for detection of latent Mycobacterium tuberculosis: a pilot study in rheumatoid arthritis. J Rheumatol 2008;35:770-5.

12. Bocchino M, Matarese A, Bellofiore B, Giacomelli P, Santoro G, Balato N, et al. Performance of two commercial blood IFN-gamma release assays for the detection of Mycobacterium tuberculosis infection in patient candidates for anti-TNF-alpha treatment. Eur J Clin Microbiol Infect Dis 2008;27:907-13.

13. Malaviya AN, Kapoor S, Garg S, Rawat R, Shankar S, Nagpal S, et al. Preventing tuberculosis flare in patients with inflammatory rheumatic diseases receiving tumor necrosis factor-alpha inhibitors in India - An audit report. J Rheumatol 2009;36:1414-20.

14. Takahashi H, Shigehara K, Yamamoto M, Suzuki C, Naishiro Y, Tamura $\mathrm{Y}$, et al. Interferon gamma assay for detecting latent tuberculosis infection in rheumatoid arthritis patients during infliximab administration. Rheumatol Int 2007;27:1143-8.

15. Greveson K, Goodhand J, Capocci S, Woodward S, Murray C, Cropley I, et al. Yield and cost effectiveness of mycobacterial infection detection using a simple IGRA-based protocol in UK subjects with inflammatory bowel disease suitable for anti-TNF $\alpha$ therapy. J Crohns Colitis 2013;7:412-8.

16. Steffen RE, Caetano R, Pinto M, Chaves D, Ferrari R, Bastos M, et al. Cost-effectiveness of Quantiferon ${ }^{\circledR}$-TB Gold-in-Tube versus tuberculin skin testing for contact screening and treatment of latent tuberculosis infection in Brazil. PLoS One 2013;8:e59546.

17. Muñoz L, Santin M. Interferon- $\gamma$ release assays versus tuberculin skin test for targeting people for tuberculosis preventive treatment: an evidence-based review. J Infect 2013;66:381-7.

18. Kim SH, Lee SO, Park JB, Park IA, Park SJ, Yun SC, et al. A prospective longitudinal study evaluating the usefulness of a T-cell-based assay for latent tuberculosis infection in kidney transplant recipients. Am J Transplant 2011;11:1927-35.

19. Hatemi G, Melikoglu M, Fresko I, Masatlioglu S, Tascilar K, Yazici $H$. Infliximab does not suppress the tuberculin skin test (purified protein derivative). J Rheumatol 2007;34:474-80.

20. Xie X, Chen JW, Li F, Tian J, Gao JS, Zhang D. A T-cell-based enzyme-linked immunospot assay for tuberculosis screening in Chinese patients with rheumatic diseases receiving infliximab therapy. Clin Exp Med 2011;11:155-61.

21. Whitworth HS, Scott M, Connell DW, Dongés B, Lalvani A IGRAs - the gateway to T cell based TB diagnosis. Methods 2013;61:52-62

J Rheumatol 2013;40:1938-40; doi:10.3899/jrheum.131218 